\title{
Model predictive control for residential energy resources using a mixed-logical dynamic model
}

\author{
R.R. Negenborn, M. Houwing, B. De Schutter, J. Hellendoorn
}

\begin{abstract}
With the increase in the number of distributed energy resources and the amount of intelligence in electricity infrastructures, the possibilities for minimizing costs of household energy consumption increase. Household systems are hybrid systems, in the sense that they exhibit both continuous and discrete dynamics. In this paper the mixed-logical dynamic framework is used to construct a dynamic model of a household system equipped with distributed energy resources. A model predictive controller (MPC) is then proposed that uses the mixed-logical dynamic model to control the energy flows inside the household. In simulation studies we assess the performance of the proposed controller, and we illustrate how additional profits can be obtained by increasing the decision freedom of the controller.
\end{abstract}

\section{INTRODUCTION}

Distributed energy resources (DER), comprising distributed power generators, energy storage units, and responsive loads, can play a crucial role in supporting many countries' key policy objectives of market liberalization, combating climate change, increasing the amount of electricity generated from renewable sources, and enhancing energy saving. Distributed generation of electricity, e.g., via photovoltaic cells, wind turbines, or combined heat and power plants, will pervade the electricity infrastructure in the future [1]. In addition, several electricity storage technologies are under development (e.g., lithium-ion batteries and plug-in hybrid electric vehicles [2]) and options for load response are foreseen for the future power system [3].

Households with distributed energy resources can operate more independently of energy suppliers and in addition they can buy and sell power to and from their suppliers. In order to determine how households can cost-efficiently manage their energy resources, we propose the use of model predictive control (MPC). Using MPC, expected energy consumption profiles, energy prices, and operational constraints can all be taken into account in determining DER technology settings and in making decisions on trading energy with suppliers. A major challenge that hereby has to be addressed is how to efficiently capture the dynamics of a household into a prediction model suitable for control. In earlier work, e.g., [4], [5], the prediction model was constructed in an ad-hoc

R.R. Negenborn (corresponding author), B. De Schutter, and J. Hellendoorn are with the Delft Center for Systems and Control, Delft University of Technology, Mekelweg 2, 2628 CD Delft, The Netherlands, $\{r . r . n e g e n b o r n, j . h e l l e n d o o r n\}$ atudelft.nl, bedeschutter.info. B. De Schutter is also with the Marine and Transport Technology department of Delft University of Technology. M. Houwing is with the Faculty of Technology, Policy and Management, Delft University of Technology, Jaffalaan 5, 2628 BX Delft, The Netherlands, m.houwingatudelft.nl.

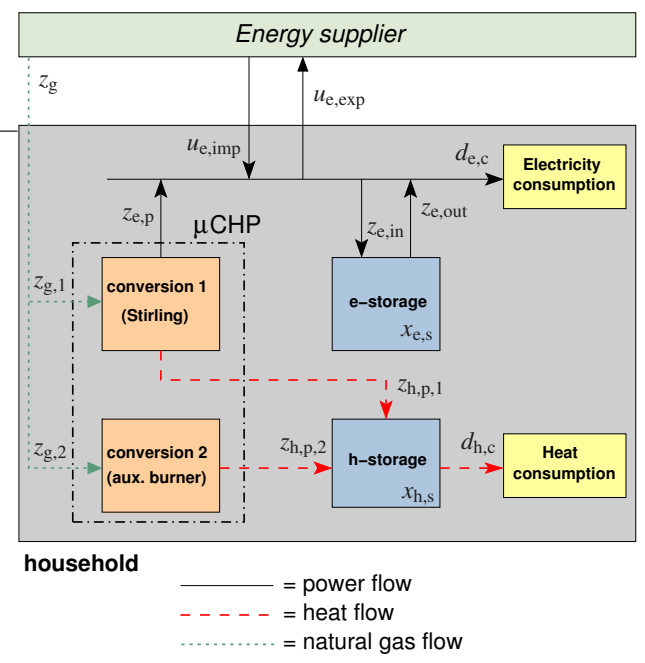

Fig. 1. Conceptual overview of the system under study [4].

fashion, not employing a formalized modeling approach. In this paper we propose the use of so-called mixed-logical dynamic models [6], which in a structured way can adequately model both the continuous and discrete dynamics present in households.

This paper is organized as follows. In Section II we introduce the system under study. The mixed-logical dynamic framework is briefly described in Section III. Next, we develop the mixed-logical dynamic model for our benchmark system in Section IV. In Section $\mathrm{V}$ we propose a model predictive controller using this mixed-logical dynamic model. Simulation experiments illustrate the performance of the proposed controller in Section VI. Section VII concludes this paper and provides directions for future research.

\section{SYSTEM DESCRIPTION}

The system under study consists of a household interacting with its energy supplier, as depicted in Figure 1. The household can buy electricity and gas from its energy supplier. In addition, the household can sell electricity to the energy supplier. The household can produce this electricity using a micro combined heat and power $(\mu \mathrm{CHP})$ unit [1]. This unit can simultaneously produce heat and electricity for the household. Heat can be stored in a heat storage unit in the form of hot water. Electricity can be stored in a battery.

We assume that the $\mu \mathrm{CHP}$ unit in the household is based on Stirling technology [1]. The $\mu \mathrm{CHP}$ unit consists of a Stirling engine prime mover, referred to as conversion unit 1 , and an auxiliary burner, referred to as conversion unit 2. Conversion unit 1 can operate in part or full load and has a 
minimum activation constraint, i.e., it has to stay in operation for a minimum amount of time before it is allowed to be switched off. For energy efficiency reasons conversion unit 2 should be used as a backup heat generator only. Therefore, priority has to be given to conversion unit 1 . Conversion units 1 and 2 are equipped with built-in on/off controllers and should produce heat in order to keep the temperature level of water in the heat storage unit between certain upper and lower bounds.

The system just described comprises hybrid dynamics, in the sense that continuously evolving dynamics are present in combination with discrete events. The continuously evolving dynamics of the energy flows and storage units changes due to discrete events caused by saturations and the logic of the built-in on/off controllers. We use mixed-logical dynamic models, as described next, to model the hybrid system.

\section{MIXED-LOGICAL DYNAMIC MODELS}

Since we are interested in model predictive control, we have to construct a suitable prediction model of the hybrid system. Discrete logic statements (e.g., statements of the form if ..., then ..., else ...) are usually part of the description of the behavior of such hybrid systems. It would be convenient if these logic statements could be transformed into linear mixed-integer constraints (i.e., constraints involving both variables that take on values from a continuous set of values, and variables that take on values from a discrete set of values), since optimization problem solvers that can deal with these constraints efficiently are available [7].

In [8], [6] it is shown how discrete logic statements can be transformed into linear mixed-integer equality and inequality constraints. As in [6], we denote continuous variables by $\mathbf{x} \in$ $\mathbb{R}^{n_{\mathbf{x}}}$ and a binary logical variable by $\delta \in\{0,1\}$. In addition, we denote a logic statement by [exp], which has as value the evaluation of an expression exp to true or false. So, $[f(\mathbf{x}) \leq 0]$ evaluates to true when $f(\mathbf{x}) \leq 0$, and to false otherwise.

Some useful transformations from logic statements into linear mixed-integer inequality constraints are given by [6]:

$$
\begin{aligned}
& {\left[\begin{array}{l}
{[f(\mathbf{x}) \leq 0] \wedge[\delta=1] \text { is true iff }} \\
f(\mathbf{x})-\delta \leq-1+\gamma_{\mathrm{m}}(1-\delta)
\end{array}\right.} \\
& {[f(\mathbf{x}) \leq 0] \vee[\delta=1] \text { is true iff } f(\mathbf{x}) \leq \gamma_{\mathrm{M}} \delta} \\
& \sim[f(\mathbf{x}) \leq 0] \text { is true iff } f(\mathbf{x}) \geq \gamma_{\varepsilon, \text { mach }} \\
& {[f(\mathbf{x}) \leq 0] \Rightarrow[\delta=1] \text { is true iff }} \\
& \qquad f(\mathbf{x}) \geq \gamma_{\varepsilon, \text { mach }}+\left(\gamma_{\mathrm{m}}-\gamma_{\varepsilon, \text { mach }}\right) \delta \\
& {[f(\mathbf{x}) \leq 0] \Leftrightarrow[\delta=1] \text { is true iff }} \\
& \qquad\left\{\begin{array}{c}
f(\mathbf{x}) \leq \gamma_{\mathrm{M}}(1-\delta) \\
f(\mathbf{x}) \geq \gamma_{\varepsilon, \text { mach }}+\left(\gamma_{\mathrm{m}}-\gamma_{\varepsilon, \text { mach }}\right) \delta
\end{array}\right.
\end{aligned}
$$

where $f: \mathscr{X} \rightarrow \mathbb{R}, \mathscr{X} \subset \mathbb{R}^{n_{\mathbf{x}}}$ is a given bounded set, $\gamma_{\varepsilon \text {, mach }}$ is a small positive constant, e.g., the machine precision, which indicates when a constraint is considered to be violated, and

$$
\gamma_{\mathrm{M}}=\max _{\mathbf{x} \in \mathscr{X}} f(\mathbf{x}), \gamma_{\mathrm{m}}=\min _{\mathbf{x} \in \mathscr{X}} f(\mathbf{x}) .
$$

Products of logical variables can be transformed into linear inequalities. E.g., the product term $\delta_{1} \delta_{2}$ can be replaced by an auxiliary binary variable $\delta_{3}$ and linear inequalities as follows [6]:

$$
\delta_{3}=\delta_{1} \delta_{2} \text { is true iff }\left\{\begin{array}{c}
-\delta_{1}+\delta_{3} \leq 0 \\
-\delta_{2}+\delta_{3} \leq 0 \\
\delta_{1}+\delta_{2}-\delta_{3} \leq 1
\end{array}\right.
$$

The product term $\delta f(\mathbf{x})$, for a function $f: \mathscr{X} \rightarrow \mathbb{R}$ and $\delta \in\{0,1\}$, is replaced by an auxiliary variable $z$ and linear inequality constraints as follows [6]:

$$
z=\delta f(\mathbf{x}) \text { is true iff }\left\{\begin{array}{c}
z \leq \gamma_{\mathrm{M}} \delta \\
z \geq \gamma_{\mathrm{m}} \delta \\
z \leq f(\mathbf{x})-\gamma_{\mathrm{m}}(1-\delta) \\
z \geq f(\mathbf{x})-\gamma_{\mathrm{M}}(1-\delta),
\end{array}\right.
$$

where $\gamma_{M}$ and $\gamma_{m}$ are as defined in (6).

For hybrid systems involving a combination of affine dynamics (on polytopic regions in the input and state space) and if ..., then ..., else ... rules, a prediction model can be cast into mixed-logical dynamic form using the transformations to obtain a compact representation of the hybrid dynamics as follows [6]:

$$
\begin{array}{r}
\mathbf{x}(k+1)=\mathbf{A x}(k)+\mathbf{B}_{1} \mathbf{u}(k)+\mathbf{B}_{2} \boldsymbol{\delta}(k)+\mathbf{B}_{3} \mathbf{z}(k) \\
\mathbf{y}(k)=\mathbf{C x}(k)+\mathbf{D}_{1} \mathbf{u}(k)+\mathbf{D}_{2} \boldsymbol{\delta}(k)+\mathbf{D}_{3} \mathbf{z}(k) \\
\mathbf{E}_{2} \boldsymbol{\delta}(k)+\mathbf{E}_{3} \mathbf{z}(k) \leq \mathbf{E}_{1} \mathbf{u}(k)+\mathbf{E}_{4} \mathbf{x}(k)+\mathbf{E}_{5},
\end{array}
$$

where

$$
\mathbf{x}(k)=\left[\begin{array}{l}
\mathbf{x}_{\mathrm{c}}(k) \\
\mathbf{x}_{\mathrm{b}}(k)
\end{array}\right] \quad \mathbf{y}(k)=\left[\begin{array}{l}
\mathbf{y}_{\mathrm{c}}(k) \\
\mathbf{y}_{\mathrm{b}}(k)
\end{array}\right] \quad \mathbf{u}(k)=\left[\begin{array}{l}
\mathbf{u}_{\mathrm{c}}(k) \\
\mathbf{u}_{\mathrm{b}}(k)
\end{array}\right],
$$

are the state, output, and input, respectively, separated into continuous components and binary components, i.e., $\mathbf{x}_{\mathrm{c}}(k) \in$ $\mathbb{R}^{n_{\mathbf{x}_{\mathrm{c}}}}, \mathbf{x}_{\mathrm{b}}(k) \in \mathbb{R}^{n_{\mathbf{x}_{\mathrm{b}}}}, n_{\mathbf{x}}=n_{\mathbf{x}_{\mathrm{c}}}+n_{\mathbf{x}_{\mathrm{b}}}, \mathbf{y}_{\mathrm{c}}(k) \in \mathbb{R}^{n_{\mathbf{y}_{\mathrm{c}}}}, \mathbf{y}_{\mathrm{b}}(k) \in \mathbb{R}^{n_{\mathbf{y}_{\mathrm{b}}}}$, $n_{\mathbf{y}}=n_{\mathbf{y}_{\mathrm{c}}}+n_{\mathbf{y}_{\mathrm{b}}}, \mathbf{u}_{\mathrm{c}}(k) \in \mathbb{R}^{n_{\mathbf{u}_{\mathrm{c}}}}, \mathbf{u}_{\mathrm{b}}(k) \in \mathbb{R}^{n_{\mathbf{u}_{\mathrm{b}}}}, n_{\mathbf{u}}=n_{\mathbf{u}_{\mathrm{c}}}+n_{\mathbf{u}_{\mathrm{b}}}$. In addition, $\boldsymbol{\delta}(k)$ are the binary variables and $\mathbf{z}(k)$ are the auxiliary continuous variables.

\section{SYSTEM DYNAMICS}

Below we formalize the dynamics of the household using the transformations of Section III to obtain a prediction model consisting of only mixed-integer linear equality and inequality constraints. In the modeling, we consider a time step length of a quarter of an hour, and for most of the variables we consider as unit the amount of energy exchanged over this quarter.

1) Conversion unit 1: Conversion unit 1 can operate at part or full load. The control inputs are therefore $u_{1 \text {,part }}(k) \in$ $\{0,1\}$ and $u_{1, \text { full }}(k) \in\{0,1\}$, where input $u_{1, \text { full }}(k)$ can only be used when $u_{1, \text { part }}(k)=1$. The energy contained in the gas used per quarter $z_{\mathrm{g}, 1}(k)$, the electricity provided to the internal network per quarter $z_{\mathrm{e}, \mathrm{p}}(k)$, and the thermal energy provided to the heat storage unit per quarter $z_{\mathrm{h}, \mathrm{p}, 1}(k)$ are given by:

$$
\begin{aligned}
z_{\mathrm{g}, 1}(k) & =\eta_{\mathrm{g}, \text { part }} u_{1, \text { part }}(k)+\left(\eta_{\mathrm{g}, \max }-\eta_{\mathrm{g}, \mathrm{part}}\right) u_{1, \mathrm{full}}(k) \\
z_{\mathrm{e}, \mathrm{p}}(k) & =\eta_{\mathrm{e}} z_{\mathrm{g}, 1}(k) \\
z_{\mathrm{h}, \mathrm{p}, 1}(k) & =\left(\eta_{\mathrm{tot}}-\eta_{\mathrm{e}}\right) z_{\mathrm{g}, 1}(k)
\end{aligned}
$$


where $\eta_{\mathrm{g}, \text { part }}$ (in $\mathrm{kWh}$ ) is the energy in the consumed gas per quarter when the conversion unit operates in part load, $\eta_{\mathrm{g}, \max }$ (in $\mathrm{kWh}$ ) is the energy in the gas consumed per quarter when the conversion unit operates in full load, $\eta_{\mathrm{e}}$ is the electric efficiency of the unit, and $\eta_{\text {tot }}$ is the total efficiency of the unit, i.e., the sum of the electric and thermal efficiencies.

The conversion unit cannot operate in full generation mode, without having it's partial generation $u_{1, \text { full }}(k)$ switched on at the same time. This behavior is modeled with the inequality constraint:

$$
u_{1, \text { full }}(k)-u_{1, \text { part }}(k) \leq 0 .
$$

Conversion unit 1 has a minimum activation constraint to avoid fast wear and tear of the device due to frequent on and off switching. The minimum activation constraint specifies that when the device has been switched on it has to stay in operation for at least $\eta_{\text {act.min }} \in \mathbb{N}^{+}$time units, with $\mathbb{N}^{+}$the set of the positive natural numbers. To model the minimum activation constraint, we introduce the counter $x_{\text {act }}(k) \in\left[0, x_{\text {act,max }}\right]$ (with $x_{\text {act,max }}$ a finite upper bound on the maximum time that a device can be in operation), which counts the number of time units that the device has been in operation so far. The evolution of this variable is given by the relation:

$$
x_{\mathrm{act}}(k+1)= \begin{cases}x_{\mathrm{act}}(k)+1 & \text { if } u_{1, \mathrm{part}}(k)=1 \\ 0 & \text { otherwise. }\end{cases}
$$

Using (8) this relation can be transformed into mixed-integer inequality constraints.

If the activation $x_{\text {act }}(k)$ is 0 , then the conversion unit is allowed to stay switched off or to be switched on. However, if the activation $x_{\text {act }}(k)$ is larger than 0 , then the conversion unit is not allowed to be switched off, until the activation $x_{\text {act }}(k)$ has reached the minimum activation $\eta_{\text {act,min }}$. Hence, as long as $x_{\text {act }}(k)$ is larger than 0 and smaller than $\eta_{\text {act,min }}$, the value of input $u_{1 \text {,part }}(k)$ should stay at its maximum, i.e., 1 . After the activation $x_{\text {act }}(k)$ has reached the minimum activation, the input $u_{1 \text {,part }}(k)$ is allowed to have a different value again. To model this, introduce a constraint on the minimum value of $u_{1, \text { part }}(k)$ as follows:

$$
u_{1, \operatorname{part}, \min }(k) \leq u_{1, \operatorname{part}}(k),
$$

with $u_{1, \text { part,min }}(k) \in\{0,1\}$. Using the activation variable $x_{\text {act }}(k)$ and this constraint we can enforce the minimum activation constraint by adjusting the lower limit $u_{1, \text { part,min }}(k)$ of $u_{1, \text { part }}(k)$ with the relation:

$$
\left[1 \leq x_{\mathrm{act}}(k) \leq \eta_{\mathrm{act}, \min }-1\right] \Leftrightarrow\left[u_{1, \mathrm{part}, \min }(k)=1\right] .
$$

To transform this relation we introduce auxiliary binary variables $\delta_{1}(k)$ and $\delta_{2}(k)$ and the following relations:

$$
\begin{aligned}
{\left[1 \leq x_{\text {act }}(k)\right] } & \Leftrightarrow\left[\delta_{1}(k)=1\right] \\
{\left[x_{\text {act }}(k) \leq \eta_{\text {act,min }}-1\right] } & \Leftrightarrow\left[\delta_{2}(k)=1\right] \\
u_{1, \text { part,min }}(k) & =\delta_{1}(k) \delta_{2}(k) .
\end{aligned}
$$

To transform these three relations into mixed-integer inequality constraints, (5) and (7) are used. With these constraints, variable $u_{1, \text { part,min }}(k)$ is constraint to the value 1 if the device should be kept in operation, and to the value 0 otherwise.

An on/off controller is installed in conversion unit 1. This on/off controller switches the conversion unit on when the amount of thermal energy in the heat storage unit $x_{\mathrm{h}, \mathrm{s}}(k)$ is lower than a lower limit $\eta_{\mathrm{h}, \mathrm{s}, \mathrm{lim}, \mathrm{min}, 1}$, and switches it off when the thermal energy in the heat storage unit $x_{\mathrm{h}, \mathrm{s}}(k)$ is larger than an upper limit $\eta_{\mathrm{h}, \mathrm{s}, \mathrm{lim}, \mathrm{max}, 1}$. In this way, the on/off controller ensures that a minimal amount of thermal energy is present in the heat storage unit. Let $u_{1 \text {,part,tmp }}(k) \in\{0,1\}$ denote the actuator setting that the on/off controller would choose if the minimum activation constraint would not be present. The on/off controller determines the value for this variable as follows:

$$
u_{1, \text { part,tmp }}(k)= \begin{cases}1 & \text { for } x_{\mathrm{h}, \mathrm{s}}(k) \leq \eta_{\mathrm{h}, \mathrm{s}, \mathrm{lim}, \min , 1} \\ 0 & \text { for } x_{\mathrm{h}, \mathrm{s}}(k) \geq \eta_{\mathrm{h}, \mathrm{s}, \mathrm{lim}, \max , 1} \\ u_{1, \text { part }}(k-1) & \text { otherwise. }\end{cases}
$$

To transform this relation auxiliary variables $\delta_{3}(k), \delta_{4}(k)$, $\delta_{5}(k)$, and $\delta_{6}(k)$ are defined such that:

$$
\begin{aligned}
{\left[\delta_{3}(k)=1\right] } & \Leftrightarrow\left[x_{\mathrm{h}, \mathrm{s}}(k) \leq \eta_{\mathrm{h}, \mathrm{s}, \mathrm{lim}, \min , 1}\right] \\
{\left[\delta_{4}(k)=1\right] } & \Leftrightarrow\left[x_{\mathrm{h}, \mathrm{s}}(k) \geq \eta_{\mathrm{h}, \mathrm{s}, \mathrm{lim}, \max , 1}\right] \\
\delta_{5}(k) & =\left(1-\delta_{3}(k)\right)\left(1-\delta_{4}(k)\right) \\
\delta_{6}(k) & =\delta_{5}(k) u_{1, \text { part }}(k-1) .
\end{aligned}
$$

Using (5) and (7) these relations are transformed into linear mixed-integer constraints. Given the values for these auxiliary variables, the on/off controller determines the value for $u_{1, \mathrm{part}, \mathrm{tmp}}(k)$ as:

$$
u_{1, \mathrm{part}, \mathrm{tmp}}(k)=1 . \delta_{3}(k)+\delta_{6}(k) .
$$

In determining the actual setting for conversion unit 1 , the on/off controller has to respect the minimum activation constraint. Therefore, the value that the on/off controller of conversion unit 1 chooses as input $u_{1, p a r t}(k)$ to the actuator of conversion unit 1 is not the value of $u_{1, \text { part,tmp }}(k)$ directly, but the value determined as follows:

$u_{1, \text { part }}(k)= \begin{cases}1 & \text { if not allowed to switch off } \\ u_{1, \text { part tmp }}(k) & \text { otherwise }\end{cases}$

which can be written as:

$u_{1, \text { part }}(k)=1 \cdot u_{1, \text { part,min }}(k)+\left(1-u_{1, \text { part,min }}(k)\right) u_{1, \text { part,tmp }}(k)$,

where $u_{1, \operatorname{part}, \min }(k)$ is defined through the minimum activation constraints (18)-(20). This relation can be transformed into linear mixed-integer constraints using (7).

2) Conversion unit 2: Conversion unit 2 has as control input $u_{2}(k) \in\left[0, u_{2, \max }\right]$. The energy in the gas used per quarter $z_{\mathrm{g}, 2}(k)$ and the thermal energy provided per quarter $z_{\mathrm{h}, \mathrm{p}, 2}(k)$ are given by:

$$
\begin{aligned}
z_{\mathrm{g}, 2}(k) & =u_{2}(k) \\
z_{\mathrm{h}, \mathrm{p}, 2}(k) & =\eta_{\mathrm{tot}} z_{\mathrm{g}, 2}(k) .
\end{aligned}
$$

A device-in-operation variable $\delta_{\text {dio, } 2}(k) \in\{0,1\}$ indicating when conversion unit 2 is in operation is defined as:

$$
\left[u_{2}(k) \geq \gamma_{\varepsilon, \text { mach }}\right] \Leftrightarrow\left[\delta_{\mathrm{dio}, 2}(k)=1\right] .
$$


This relation can be converted into linear mixed-integer inequality constraints using (5). The device-in-operation variable $\delta_{\text {dio,2 }}(k)$ is used to enforce that conversion unit 2 is in operation only when conversion unit 1 is in operation through the following constraints:

$$
\delta_{\text {dio }, 2}(k)-u_{1, \text { part }}(k) \leq 0 .
$$

A on/off controller is installed in conversion unit 2, similar to the on/off controller as in conversion unit 1 . The on/off controller of conversion unit 2 determines an auxiliary actuator setting $u_{2, \mathrm{tmp}}(k) \in\{0,1\}$ as follows:

$$
u_{2, \mathrm{tmp}}(k)= \begin{cases}1 & \text { for } x_{\mathrm{h}, \mathrm{s}}(k) \leq \eta_{\mathrm{h}, \mathrm{s}, \mathrm{lim}, \min , 2} \\ 0 & \text { for } x_{\mathrm{h}, \mathrm{s}}(k) \geq \eta_{\mathrm{h}, \mathrm{s}, \mathrm{lim}, \max , 2} \\ u_{2, \mathrm{tmp}}(k-1) & \text { otherwise }\end{cases}
$$

To transform this relation, auxiliary variables $\delta_{7}(k), \delta_{8}(k)$, $\delta_{9}(k)$, and $\delta_{10}(k)$ are defined such that:

$$
\begin{aligned}
{\left[\delta_{7}(k)=1\right] } & \Leftrightarrow\left[x_{\mathrm{h}, \mathrm{s}}(k) \leq \eta_{\mathrm{h}, \mathrm{s}, \mathrm{lim}, \min , 2}\right] \\
{\left[\delta_{8}(k)=1\right] } & \Leftrightarrow\left[x_{\mathrm{h}, \mathrm{s}}(k) \geq \eta_{\mathrm{h}, \mathrm{s}, \lim , \max , 2]}\right] \\
\delta_{9}(k) & =\left(1-\delta_{7}(k)\right)\left(1-\delta_{8}(k)\right) \\
\delta_{10}(k) & =\delta_{9}(k) u_{2, \mathrm{tmp}}(k-1) .
\end{aligned}
$$

Using (5) and (7) these relations are transformed into linear mixed-integer constraints. The on/off controller determines the value for the auxiliary actuator setting $u_{2, \operatorname{tmp}}(k)$ as:

$$
u_{2, \mathrm{tmp}}(k)=1 . \delta_{7}(k)+\delta_{10}(k)
$$

The on/off controller uses this auxiliary actuator setting to determine the actual input for conversion unit 2 as:

$$
u_{2}(k)=u_{2, \mathrm{tmp}}(k) \eta_{\mathrm{frac}} u_{2, \max }
$$

where $\eta_{\text {frac }}$ is the part of the maximum output $u_{2 \text {, max }}$ that is activated when conversion unit 2 is switched on.

3) Electricity and heat storage units: The dynamics of the electrical energy in the storage unit per quarter $x_{\mathrm{e}, \mathrm{s}}(k)$ are given by:

$$
x_{\mathrm{e}, \mathrm{s}}(k+1)=x_{\mathrm{e}, \mathrm{s}}(k)+z_{\mathrm{e}, \text { in }}(k)-z_{\mathrm{e}, \text { out }}(k),
$$

where $z_{\mathrm{e}, \text { in }}(k)$ and $z_{\mathrm{e}, \text { out }}(k)$ are the electrical energy going into and taken from the storage unit per quarter, respectively, and where it is assumed that the charging and discharging of the battery takes place without energy loss.

The dynamics of the thermal energy in the heat storage unit per quarter $x_{\mathrm{h}, \mathrm{s}}(k)$ are given by:

$$
x_{\mathrm{h}, \mathrm{s}}(k+1)=x_{\mathrm{h}, \mathrm{s}}(k)+z_{\mathrm{h}, \mathrm{p}, 1}(k)+z_{\mathrm{h}, \mathrm{p}, 2}(k)-d_{\mathrm{h}, \mathrm{c}}(k),
$$

where $d_{\mathrm{h}, \mathrm{c}}(k)$ is the heat consumption per quarter.

The electrical and thermal energy levels of the storage units are limited by minimum and maximum values, i.e.:

$$
\begin{aligned}
& x_{\mathrm{e}, \mathrm{s}, \min } \leq x_{\mathrm{e}, \mathrm{s}}(k) \leq x_{\mathrm{e}, \mathrm{s}, \max } \\
& x_{\mathrm{h}, \mathrm{s}, \min } \leq x_{\mathrm{h}, \mathrm{s}}(k) \leq x_{\mathrm{h}, \mathrm{s}, \max } .
\end{aligned}
$$

4) Power balance: The following power balance relating the amount of electricity generated per quarter by conversion unit $1 z_{\mathrm{e}, \mathrm{p}}(k)$, the electricity input to the battery per quarter $z_{\mathrm{e}, \text { in }}(k)$, the electricity output from the battery per quarter $z_{\mathrm{e}, \text { out }}(k)$, the electricity consumption per quarter $d_{\mathrm{e}, \mathrm{c}}(k)$, and the electricity bought from $u_{\mathrm{e}, \mathrm{imp}}(k)$ or sold to $u_{\mathrm{e}, \exp }(k)$ the energy supplier per quarter, has to hold:

$$
\begin{aligned}
0=z_{\mathrm{e}, \mathrm{p}}(k)+u_{\mathrm{e}, \mathrm{imp}}(k)+z_{\mathrm{e}, \text { out }}( & (k)-u_{\mathrm{e}, \exp }(k) \\
& -z_{\mathrm{e}, \text { in }}(k)-d_{\mathrm{e}, \mathrm{c}}(k) .
\end{aligned}
$$

All of this results in an MLD model of the form (9)-(11).

\section{MPC PROBLEM FORMULATION}

We now use the derived model as prediction model for a controller controlling the energy flows of a household. The controller uses an MPC strategy such that the controller can:

- optimize the usage of the storage units;

- take into account the decision freedom due to the possibility of electricity import, export, and generation;

- incorporate predictions on electricity and heat consumption;

- incorporate models of the dynamics and constraints of installed generators and storage units,

while maintaining the amount of energy in the heat storage unit between a desired upper and lower limit, and respecting the operational constraints, including a minimal activation of 2 time units for conversion unit 1 .

\section{A. MPC scheme}

At each control step $k$ the controller makes a measurement of the system state consisting of values for $x_{\mathrm{h}, \mathrm{s}}(k), x_{\mathrm{e}, \mathrm{s}}(k)$, and $x_{\text {act }}(k)$. Then the controller determines values for the control inputs $u_{1, \text { full }}(k), u_{\mathrm{e}, \text { imp }}(k)$, and $u_{\mathrm{e}, \exp }(k)$ by solving the MPC optimization problem that minimizes a cost function, subject to the prediction model, operational constraints, and initial state. With respect to the conversion units, the controller only determines $u_{1 \text {,full }}(k)$, since $u_{1 \text {,part }}(k)$ and $u_{2}(k)$ are determined by the on/off controllers.

1) Cost function: The main objective of the controller is to minimize the daily operational costs of residential energy use. These costs depend on the price $p_{\mathrm{f}}(€ / \mathrm{kWh})$ for gas consumption, the price $p_{\text {imp }}(k)(€ / \mathrm{kWh})$ at which electricity can be bought, and the price $p_{\exp }(€ / \mathrm{kWh})$ at which electricity can be sold. In principle, the prices for gas, electricity import, and electricity output vary over the day. However, as a first step we assume that the $p_{\mathrm{f}}$ and $p_{\exp }$ are constant, whereas the $p_{\text {imp }}$ varies over the day.

In addition to minimizing the daily operational cost, the controller should maintain $x_{\mathrm{h}, \mathrm{s}}(k)$ between the desired upper and lower limits. This goal is included as a soft constraint by penalizing an auxiliary variable $z_{\text {aux }}(k+l) \geq 0$, for $l \in$ $\{1, \ldots, N\}$, with a large positive cost $p_{\text {soft }}$. This auxiliary variable $z_{\text {aux }}(k+l)$ is defined such that:

$z_{\text {aux }}(k+l)=$

$$
\begin{cases}x_{\mathrm{h}, \mathrm{s}}(k+l)-\eta_{\mathrm{h}, \mathrm{s}, \mathrm{lim}, \max } & \text { for } x_{\mathrm{h}, \mathrm{s}}(k+l) \geq \eta_{\mathrm{h}, \mathrm{s}, \text { lim,max }} \\ \eta_{\mathrm{h}, \mathrm{s}, \mathrm{lim}, \min }-x_{\mathrm{h}, \mathrm{s}}(k+l) & \text { for } x_{\mathrm{h}, \mathrm{s}}(k+l) \leq \eta_{\mathrm{h}, \mathrm{s}, \text { lim,min }} \\ 0 & \text { otherwise }\end{cases}
$$


TABLE I

VALUES OF THE PARAMETERS OF THE HOUSEHOLD SYSTEM.

\begin{tabular}{ccl|ccl}
\hline parameter & {$[\mathrm{unit}]$} & value & parameter & {$[\mathrm{unit}]$} & value \\
\hline$u_{2, \max }$ & {$[\mathrm{kWh}]$} & 4.9383 & $\eta_{\mathrm{g}, \max }$ & {$[\mathrm{kWh}]$} & 1.8333 \\
$x_{\mathrm{act}, \max }$ & {$[\mathrm{steps}]$} & $1.10^{6}$ & $\eta_{\mathrm{g}, \text { part }}$ & {$[\mathrm{kWh}]$} & 0.9167 \\
$x_{\mathrm{e}, \mathrm{s}, \max }$ & {$[\mathrm{kWh}]$} & 2 & $\eta_{\text {tot }}$ & {$[-]$} & 1.0125 \\
$x_{\mathrm{e}, \mathrm{s}, \min }$ & {$[\mathrm{kWh}]$} & 0 & $\eta_{\mathrm{h}, \mathrm{s}, \mathrm{lim}, \max }$ & {$[\mathrm{kWh}]$} & 8.1278 \\
$x_{\mathrm{h}, \mathrm{s}, \max }$ & {$[\mathrm{kWh}]$} & 9.1728 & $\eta_{\mathrm{h}, \mathrm{s}, \mathrm{lim}, \max , 1}$ & {$[\mathrm{kWh}]$} & 6.9667 \\
$x_{\mathrm{h}, \mathrm{s}, \min }$ & {$[\mathrm{kWh}]$} & 0 & $\eta_{\mathrm{h}, \mathrm{s}, \text { lim,max }, 2}$ & {$[\mathrm{kWh}]$} & 5.2250 \\
$\gamma_{\varepsilon, \operatorname{mach}}$ & {$[-]$} & $1.10^{-8}$ & $\eta_{\mathrm{h}, \mathrm{s}, \text { lim,min }}$ & {$[\mathrm{kWh}]$} & 2.3222 \\
$\eta_{\mathrm{act}, \min }$ & {$[\mathrm{steps}]$} & 2 & $\eta_{\mathrm{h}, \mathrm{s}, \text { lim,min,1 }}$ & {$[\mathrm{kWh}]$} & 4.0639 \\
$\eta_{\mathrm{e}}$ & {$[-]$} & 0.15 & $\eta_{\mathrm{h}, \mathrm{s}, \text { lim,min, }}$ & {$[\mathrm{kWh}]$} & 2.9028 \\
$\eta_{\mathrm{frac}}$ & {$[-]$} & 0.6 & & &
\end{tabular}

which in combination with the minimization of the term $p_{\text {soft }} z_{\text {aux }}(k+l)$ can also be written as:

$$
\begin{aligned}
& z_{\mathrm{aux}}(k+l)= \\
& \left\{\begin{array}{l}
\eta_{\mathrm{h}, \mathrm{s}, \text { lim,min }}-z_{\mathrm{aux}}(k+l) \leq x_{\mathrm{h}, \mathrm{s}}(k+l) \\
x_{\mathrm{h}, \mathrm{s}}(k+l) \leq \eta_{\mathrm{h}, \mathrm{s}, \text { lim, } \max }+z_{\mathrm{aux}}(k+l) .
\end{array}\right.
\end{aligned}
$$

The cost function at control step $k$ over a prediction horizon of $N$ control cycles, including the costs for the soft constraints, is defined as:

$$
\begin{aligned}
J= & \sum_{l=0}^{N-1}\left(p_{\mathrm{f}}\left(z_{\mathrm{g}, 1}(k+l)+z_{\mathrm{g}, 2}(k+l)\right)-p_{\exp } u_{\mathrm{e}, \exp }(k+l)\right. \\
& \left.+p_{\text {imp }}(k+l) u_{\mathrm{e}, \text { imp }}(k+l)+p_{\text {soft }} z_{\text {aux }}(k+1+l)\right)
\end{aligned}
$$

2) Prediction model: The prediction model that the controller uses is based on the relations as given in Section II, specified over the prediction horizon. Hence, the prediction model consists of a large system of linear mixed-integer equality and inequality constraints. The values of the parameters are given in Table I.

3) Initial constraints: At control cycle $k$, the following variables are constrained to the known or measured values: $x_{\mathrm{e}, \mathrm{s}}(k), x_{\mathrm{h}, \mathrm{s}}(k), x_{\mathrm{act}}(k), u_{1, \mathrm{part}}(k-1)$, and $u_{2, \mathrm{tmp}}(k-1)$.

4) Solving the optimization problem: The MPC optimization problem is a mixed-integer linear programming problem. It is linear, since the cost function and all constraints are linear and it is mixed integer, since the problem involves continuous and discrete variables.

\section{Simulations}

To illustrate the operation of the proposed controller, we perform experiments for a particular winter day, January 29, 2006. For this day, average residential electricity and aggregated heat demand profiles have been created with 2006 data from 'EnergieNed', the Dutch Federation of Energy Companies. The controller determines new actions every 15 minutes by solving its MPC problem at that time. To set up the MPC problem prices for electricity import, electricity export, and gas consumption have to be calculated first.

\section{A. Price calculation}

The variable electricity import price $p_{\text {imp }}(k)$ is calculated as follows. The Dutch Central Bureau of Statistics states a total electricity tariff for small consumers for 2006 of $194 € / \mathrm{MWh}^{1}$ (household class: single tariff, $3000 \mathrm{kWh}$ ). The

\footnotetext{
${ }^{1}$ http://www.cbs.nl/, Dutch central bureau of statistics.
}

variable part of the total tariff (including energy and VAT taxes) is around $90 \%$ of the total tariff at the time of performing the experiments ${ }^{2}$, so this becomes $0.1746 € / \mathrm{kWh}$. The variable supply part of the total tariff accounts for $32 \%$ of the total tariff at the time of performing the experiments ${ }^{1}$. For this variable supply part we have substituted values obtained from the Amsterdam Power Exchange. For the value of the feedback tariff $p_{\exp }$ we have taken average "EnergieNed" data for 2006, which gives $0.0601 € / \mathrm{kWh}$.

The gas price $p_{\mathrm{f}}$ is determined as follows. At the website of the Dutch Central Bureau of Statistics, a total gas tariff for small consumers of $552 € / 1000 \mathrm{~m}^{3}$ is given (for consumer class: $2000 \mathrm{~m}^{3}$ ). According to the website of the Energy Research Center of The Netherlands ${ }^{2}, 91 \%$ of the gas tariff is variable (including taxes) at the time of performing the experiments. This leads to a gas price of $0.50232 € / \mathrm{m}^{3}$.

\section{B. Simulations}

To illustrate the operation of the proposed approach, we first consider a prediction horizon with length $N=16$. After that we consider the performance of the controller under varying $N$. The starting values at $k=1$ are taken as:

$$
\begin{aligned}
& x_{\mathrm{e}, \mathrm{s}}(k)=0, x_{\mathrm{h}, \mathrm{s}}(k)=5.806, x_{\mathrm{act}}(k)=0 \\
& u_{1, \mathrm{part}}(k-1)=0, u_{2, \mathrm{tmp}}(k-1)=0 .
\end{aligned}
$$

For solving the MPC problem at each control cycle the controller uses the ILOG CPLEX v10.0 linear mixed-integer programming solver through the Tomlab v5.7 interface in Matlab v7.3.

\section{Results for MPC with $N=16$}

Figure 2(a) shows the energy level in the heat storage unit each quarter for the controlled case. Figure 2(b) shows the energy consumed in the form of gas per quarter by the conversion units for the controlled case. The on/off controllers should switch on the conversion units depending on the energy content of the heat storage unit. It is observed that, indeed, when the energy content of the heat storage unit reaches one of the lower limits, the respective conversion unit is switched on, whereas when the content reaches one of the upper limits, the respective conversion unit is switched off. Conversion unit 1 is switched on 5 times throughout the day, and stays in operation at least 2 time units. Hence, the constraints on the minimal activation time of 2 time units is respected. Conversion unit 1 is switched to full load operation several times throughout the day. When this happens, the controller has ensured that conversion unit 1 is already operating at part load.

\section{Results for varying prediction horizon lengths}

For varying prediction horizon lengths, we consider two scenarios: the scenario considered so far, i.e., the scenario in which the MPC controller controls the household including on/off controllers in the conversion units, and a scenario in which the on/off controllers in the conversion units are

\footnotetext{
${ }^{2}$ http://www.energie.nl/, Energy Research Center of The Netherlands.
} 


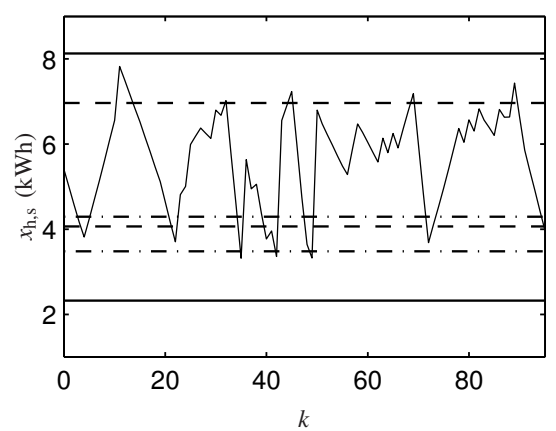

(a)

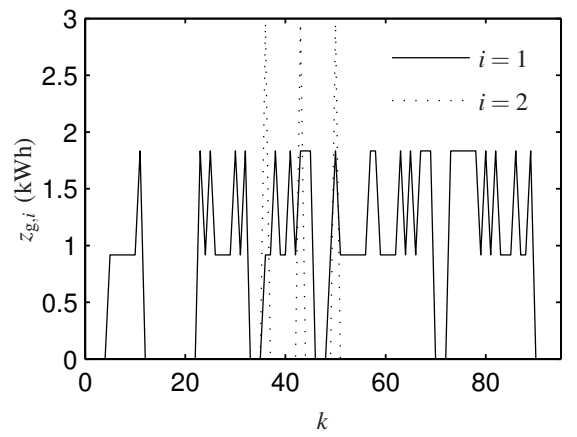

(b)

Fig. 2. (a) The energy level in the heat storage unit $x_{\mathrm{h}, \mathrm{s}}(k)$ each quarter. The dashed and dashed-dotted lines indicate upper and lower activation bounds of the on/off controllers of conversion unit 1 and 2, respectively. The solid horizontal lines indicate physical upper and lower bounds; (b) amount of energy consumed in the form of gas per quarter by the conversion units.

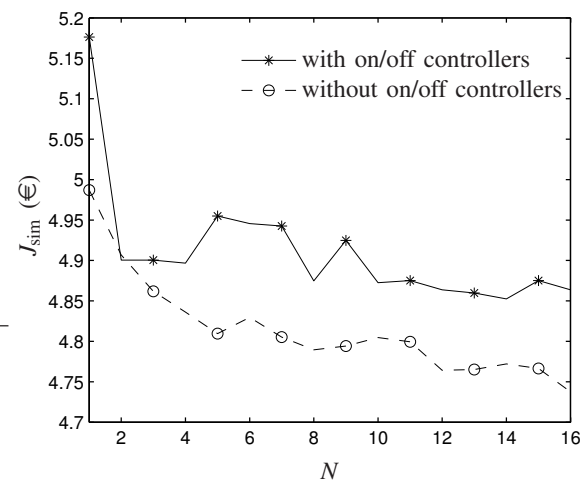

Fig. 3. Closed-loop cost $J_{\text {sim }}$ for varying prediction horizon lengths $N$, both for the scenario in which the on/off controllers is installed, and for the scenario in which the on/off controllers are not installed.

not present. In this second scenario, the MPC controller has more decision freedom, since it can determine by itself when conversion unit 1 and conversion unit 2 should be switched on or off (although it still has to respect the priority constraint).

Figure 3 shows the cost $J_{\text {sim }}$ defined over the full simulation period for varying prediction horizon lengths ${ }^{3}$. For both scenarios, there is a general trend that as the prediction horizon length increases, the performance increases as well. However, since the controller does not take into account the

\footnotetext{
${ }^{3}$ In order to make a fair comparison of the control performance for varying horizon lengths, the prediction horizon length is decreased as soon as during a simulation predictions would go over the actual simulation time span.
}

energy consumption patterns and electricity price fluctuations after its prediction horizon, it can choose actions that are not optimal over the full simulation. Therefore, in our case it is not strictly necessary that the performance increases with a longer prediction horizon. We also observe this in Figure 3. From the figure we also observe that if the on/off controllers are not present, indeed, the MPC controller can exploit the increased decision freedom. This results in a higher performance for the scenario in which the on/off controllers are not installed.

\section{CONCLUSIONS \& FUTURE RESEARCH}

In this paper we have applied the mixed-logical framework to model a household system. We have derived a model for a household equipped with its own power generation (via a micro combined heat and power unit), energy storage capabilities (via a water tank and a battery), and the possibility for energy exchange with an external energy supplier. Using this model, we have constructed a model predictive control (MPC) strategy for a controller in a household. In simulations we have illustrated the performance gain of the proposed approach that is obtained when the decision freedom of the MPC controller is increased by removing the internal on/off controller logic.

Future research consists of validating, and if necessary improving, the household model, investigating the predictability of the energy patterns of individual and groups of households, and extending the MPC approach to a distributed control setting in which households can also exchange or sell power amongst one another.

\section{ACKNOWLEDGMENTS}

Research supported by the BSIK project "Next Generation Infrastructures (NGI)", the Delft Research Center Next Generation Infrastructures, the European STREP project "Hierarchical and distributed model predictive control (HD-MPC)", and the project "Multi-Agent Control of Large-Scale Hybrid Systems" (DWV.6188) of the Dutch Technology Foundation STW.

\section{REFERENCES}

[1] M. Pehnt, M. Cames, C. Fischer, B. Praetorius, L. Schneider, K. Schumacher, and J. Vob, Micro Cogeneration: Towards Decentralized Energy Systems. Berlin, Germany: Springer, 2006.

[2] E. Lysen, S. Van Egmond, and S. Hagedoorn, "Opslag van elektriciteit: Status en toekomstperspectief voor Nederland," Utrecht Centrum voor Energieonderzoek - SenterNovem, Utrecht, The Netherlands, Tech. Rep. NEO 0268-05-05-01-002, 2006, in Dutch.

[3] S. D. Braithwait, "Real-time pricing and demand response can work within limits," Natural Gas and Electricity, vol. 21, no. 11, pp. 1-9, 2005.

[4] M. Houwing, R. R. Negenborn, P. Heijnen, B. De Schutter, and J. Hellendoorn, "Least-cost model predictive control of residential energy resources when applying $\mu \mathrm{CHP}$," in Proceedings of Power Tech 2007, Lausanne, Switzerland, July 2007, paper 291.

[5] R. R. Negenborn, M. Houwing, B. De Schutter, and J. Hellendoorn, "Adaptive prediction model accuracy in the control of residential energy resources," in Proceedings of the 2008 IEEE Multi-conference on Systems and Control, San Antonio, Texas, Sept. 2008, pp. 311-316.

[6] A. Bemporad and M. Morari, "Control of systems integrating logic, dynamics, and constraints," Automatica, vol. 35, no. 3, pp. 407-427, Mar. 1999

[7] R. Fletcher and S. Leyffer, "Numerical experience with lower bounds for MIQP branch-and-bound," SIAM Journal on Optimization, vol. 8, no. 2, pp. 604-616, May 1998.

[8] H. P. Williams, Model Building in Mathematical Programming. New York, New York: Wiley, 1993. 